\title{
Estudio Preliminar de Patrones de Mordedura según Forma del Cráneo, Mediante el Análisis Morfológico y Morfométrico de Semiarcadas Dentarias de Perro Doméstico (Canis familiaris) con Fines de Identificación
}

\author{
Preliminary Study of Bite Patterns According to Skull Shape, by Morphological and \\ Morphometric Analysis of Dental Semi Arcs of Domestic Dog (Canis familiaris) for identification \\ "Víctor Toledo González; "Luis Ibarra M.; *Valeria Rojas E.; \\ *** Luis Ciocca G.; *Nicolás Rocha D. \& *Gabriel Jara V.
}

TOLEDO, G. V.; IBARRA, M. L.; ROJAS, E. V.; CIOCCA, G. L.; ROCHA, D. N. \& JARA, V. G. Estudio preliminar de patrones de mordedura según forma del cráneo, mediante el análisis morfológico y morfométrico de semiarcadas dentarias de perro doméstico (Canis familiaris) con fines de identificación. Int. J. Morphol., 30(1):222-229, 2012.

RESUMEN: La Odontología forense es una rama de las Ciencias Forenses que se utiliza para establecer la identidad de una víctima o victimario mediante el estudio de las huellas de mordeduras, estimación de edad cronológica, entre otros. Dada la inexistencia de este tipo de estudios en Chile y la magnitud que alcanza el problema de mordidas de perros en Chile, se compararon los patrones de huellas de mordedura según forma del cráneo, mediante el análisis morfológico y morfométrico de semiarcadas dentarias de perro doméstico (Canis familiaris) con fines médico legal. Para ello se obtuvieron modelos dentarios en yeso e impresiones de huellas de mordedura en cera de tres razas puras: Bóxer, Dálmata y Ovejero Alemán. Las mediciones contemplaron la distancia máxima externa entre caninos superiores e inferiores y la distancia máxima externa entre incisivos superiores e inferiores. Los principales resultados muestran que sólo la distancia máxima entre caninos superiores permitiría identificar la raza en forma estadísticamente significativa $(\mathrm{p} \leq 0,05)$ en los modelos de yeso. En el estudio ciego y desde el punto de vista morfométrico se identificaron especialmente los Dálmata con un alto grado de sensibilidad y especificidad. Un poco menor y en forma variable se observó en Bóxer y Ovejero Alemán. El estudio morfológico permitió identificar e individualizar al 100\% de los perros. Por tanto los métodos morfométricos y morfológicos son herramientas complementarias para identificar e individualizar a un posible perro agresor.

PALABRAS CLAVE: Mordeduras de perros; Huellas de mordedura; Veterinaria forense.

\section{INTRODUCCIÓN}

En la actualidad los animales de compañía, como son el perro y el gato, no sólo adquieren un valor funcional (cuidado, trabajo, etc.), como lo fue por décadas, para el núcleo familiar y social. Hoy, el valor de estos animales para el humano incluye entre otros, aspectos afectivos, emocionales, psicológicos, señalando que estos nuevos atributos son asignados por la sociedad, con evidentes beneficios personales. Sin embargo, el aumento en el grado de integración de éstos en la vida familiar reviste un proceso de adaptación entre ambas especies. Con el aumento proporcional de perros en los hogares se ha descrito, a la vez, un aumento en el porcentaje de agresiones de diversa magnitud de éstos y/o muertes, tanto a personas, como a otros animales de compa- ñía. Muchas de las evidencias provocadas por perros, encontradas en los sitios de suceso (SS) (dientes, mordeduras, secreciones, etc.), no son consideradas en su gran mayoría en un proceso judicial en Chile, o bien, esas evidencias son analizadas por legistas sin formación veterinaria, los cuales basan su estudio en una recolección de información forense, no estandarizada a la realidad local, escasa e incluso inexistente, lo que se traduce finalmente en un proceso de investigación ineficiente e incompleto. De tal manera que se hace imprescindible contar con una base de datos de huellas de mordeduras de perros existentes en Chile, con la cual comparar aquellas presentes en un SS, permitiendo, al menos, identificar la raza del agresor.

\footnotetext{
* Facultad de Ciencias Veterinarias y Pecuarias, Universidad de Chile, Santiago, Chile.

*** Departamento Medicina Legal. Facultad de Medicina. Universidad de Chile, Santiago, Chile.

Patrocinio: Fondo de Investigación Veterinaria: FIV: 12101401.9102.007
} 
TOLEDO, G. V.; IBARRA, M. L.; ROJAS, E. V.; CIOCCA, G. L.; ROCHA, D. N. \& JARA, V. G. Estudio preliminar de patrones de mordedura según forma del cráneo, mediante el análisis morfológico y morfométrico de semiarcadas dentarias de perro doméstico (Canis familiaris) con fines de identificación. Int. J. Morphol., 30(1):222-229, 2012.

Entre las mordeduras causadas por animales, las de los perros son probablemente, las más frecuentes. Son heridas contusas y casi siempre con desgarramiento. Se diferencian de una mordida humana porque; los caninos dejan profundas huellas cónicas, la arcada dentaria es más estrecha y la impronta dejada toma una forma similar a una "V" invertida. Cuando la agresión del perro corresponde a un estado de excitación del mismo, su mordedura reviste una violencia inusitada, empleando las arcadas dentarias en su totalidad, originando siempre una mordedura de magnitud proporcional al tamaño y temperamento del animal, efectuando un mecanismo de arrancamiento, produciendo lesiones a colgajo, o de arrancamiento total (Ciocca, 2010). La apariencia macroscópica de una mordedura aguda revela una combinación de abrasiones, contusiones, en algunos casos laceraciones por ruptura de la piel o hemorragias capilares intradérmicas; variables por el sitio, tipo de piel, grosor y estado de salud, por la posición, ropa interpuesta, fuerzas y dinámicas. Todas ellas alteran el tipo de apariencia de la injuria y las reacciones de las heridas (Gaensslen, 2005).

Las fuerzas de las fauces caninas varían de acuerdo a la raza (Teke, 2001). La presión masticatoria en la zona de los molares es del orden de hasta $3.000 \mathrm{~kg} / \mathrm{cm}^{2}$, si bien difiere según raza, edad, sexo, alimento acostumbrado, etc. En los perros adiestrados para defensa y ataque, se producen presiones de alrededor de los $500 \mathrm{~kg} / \mathrm{cm}^{2}$ (Manns, 1988).

Las mordeduras y daños realizados por perros, en algunos casos fatales, son un problema mundial (Morgan, 2004) y particularmente que afecta a niños. En Chile, según los datos registrados por el Departamento de Estadística del Servicio Médico Legal (SML) en el período comprendido entre el año 1999 y 2008 el número de muertes por esta causa asciende a 7 casos mientras que el número de lesiones no mortales se estima en un promedio de 104 por año entre los años 2004 y 2008. De acuerdo a información del Departamento de Estadística del Instituto de Salud (DEIS) se registran consultas por esta causa, a servicios de salud sólo de la Región Metropolitana, de alrededor de 7.000 casos que necesitan atención médica. Por otro lado, se señala que la tasa promedio de mordeduras en Santiago es de 1.262 mordidas por cada 100 mil habitantes al año (Ibarra et al., 2003).

Las huellas de mordida o mordedura se definen como la impresión en negativo de las piezas dentarias (todas o algunas), sobre una superficie capaz de sostenerla, pudiendo ser éste un elemento inerte o biológico, como la piel humana (Teke). Esta impresión se produce mediante un mecanismo de presión y tracción (Ciocca, 1987).

Los dientes como herramientas de corte y aprehensión deben ser aceptados como marcadores de huellas de mordida en ese contexto. La dentición, como las huellas digitales, es única para cada individuo. Basta considerar para ello las variaciones en: tamaño, posición, desgaste, fracturas, ubicación en la arcada, diastema, restauraciones, entre otras (Gaensslen). Cabe señalar que un patrón de injuria, tal como una huella de mordida, no se hace visible mediante una transferencia de material y tampoco a menudo por las hendiduras, sino más bien por una respuesta vital de los tejidos mordidos. Es el raspado superficial del epitelio por el contacto con los dientes (abrasión), el sangrado dentro de la piel por la presión de los dientes (contusión), o el desgarro de la piel por los dientes (laceración), que hace visible la marca de mordedura (Gaensslen).

Cada vez con mayor frecuencia las mordeduras derivan en procesos de litigación. Como se ha informado, las principales víctimas de las mordeduras caninas son los niños (Palacio et al., 2005; Ibarra et al.) Los tramos de edad más afectados varían según los estudios entre 0-15, <6, 5-9, 7-12 y 7-9 años. Las principales víctimas de las mordeduras de perros con desenlace mortal son igualmente niños, sobre todo los más pequeños. La tasa de muerte en neonatos y bebés es significativamente superior a la de los adultos. Asimismo es frecuente la muerte de personas muy mayores, posiblemente por la dificultad de defenderse y las características generales de estos grupos de edad. Además, las principales consecuencias que derivan de las mordeduras de perros son las lesiones y cicatrices producidas por las agresiones. Además de la potencial transmisión de enfermedades infecciosas, se incluyen secuelas psicológicas, incapacidades, costos económicos derivados de los tratamientos médicos y psicológicos, bajas laborales y las más importante de todas, la muerte de la víctima, ya sea causada directamente por las lesiones producidas por la agresión o alguna de las enfermedades transmitidas a través de la mordedura (Palacio et al.). La mayoría de los ataques por perros se producen, aparentemente, sin provocación. Se alteran por ser molestados mientras comen y no gustan de ser amenazados o que su territorio se invada (Morgan \& Palmer, 2007). Muchas revisiones concluyen que el riesgo más alto está dado por perros de raza grande como el Ovejero Alemán, Pitbull Terrier, Rottweiler y Chow, pero todos los perros deberían considerarse peligrosos; incluso perros más pequeños como el Jack Russell Terrier, que puede inflingir severas mordeduras (Morgan \& Palmer).

En general, los métodos más comunes para la determinación de huellas de mordedura humana incluyen técnicas para comparar la morfología de la dentición (forma, tamaño y posición de los dientes, junto con la forma de los arcos dentales), con rasgos y características similares presentes en fotografías tamaño natural de las lesiones, con superposiciones transparentes o computacionales (Sweet et 
al., 1997; Sweet \& Bowers, 1998), entre otros (Rawson et al., 1979; David, 1986; Jakobsen et al., 1995; Bowers \& Bell, 1995; McNamee et al., 2005; Drinnan \& Melton, 1985).

Dada la existencia de un gran número de especies de animales, existentes en Chile potencialmente mordedores, y considerando que el perro doméstico se ve involucrado en el mayor número de casos con mordeduras asociadas (leves o graves), en el presente artículo se compararon algunos aspectos morfológicos y morfométricos de la dentición de ellos, que ayuden a identificar y, en lo posible, individualizar a un potencial perro agresor, mediante las huellas de mordeduras encontradas en SS.

\section{MATERIAL Y MÉTODO}

Para este estudio se constituyeron 3 grupos de perros según forma de cráneo. Grupo 1.- braquicéfalicos (Bóxer (B)); Grupo 2.- mesocefálicos (Dálmata (D)); y Grupo 3.dolicocefálicos (Ovejero Alemán (OA)). Cada grupo fue conformado por 10 ejemplares, cinco machos y cinco hembras, con dentición completa definitiva (mayores a 6 meses), sin tratamientos dentales y pertenecientes a la raza establecida (sin mestizaje). Cabe señalar que las mordeduras son efectuadas en un mayor porcentaje por dientes incisivos y caninos. Por esta razón y para realizar mediciones y comparaciones fue necesario obtener moldes de yeso de la semiarcada superior e inferior de cada uno de ellos mediante técnica y materiales utilizados en odontología (Anusavice, 2004). En los moldes se realizaron las siguientes mediciones morfométricas para su análisis comparativo mediante un caliper (Control Company @) y las medidas registradas en milímetros: Distancia máxima externa entre caninos superiores (DMCS) e inferiores (DMCI); Distancia máxima entre incisivos superiores (DMIS) e inferiores (DMII). Para la obtención de huellas de mordedura se utilizó una lámina de cera rosada dental de $1 \mathrm{~mm}$ de grosor y de $70 \mathrm{~mm}$ de ancho por $140 \mathrm{~mm}$ de largo cubriendo (por ambos lados) un bloque de poliuretano expandible de $2 \mathrm{~cm}$ de altura, sobre la cual cada ejemplar debió morder hasta la base de sus dientes caninos e incisivos permitiendo obtener las improntas de la semiarcada superior e inferior. Para resaltar los bordes de las mordeduras se pintó la cera con betún negro. Posteriormente se realizó un registro digital, mediante escáner de la huella en la cera almacenándola en la memoria del computador, generando un registro indeleble. Las imágenes fueron almacenadas en proporción 1:1 (escala real), evitando cualquier distorsión que alterara las mediciones. El estudio métrico y morfológico de las huellas se realizó mediante programa Photoshop 5,5 ®. En estas muestras se realizaron las mismas medidas que en los modelos de yeso (Fig. 1).

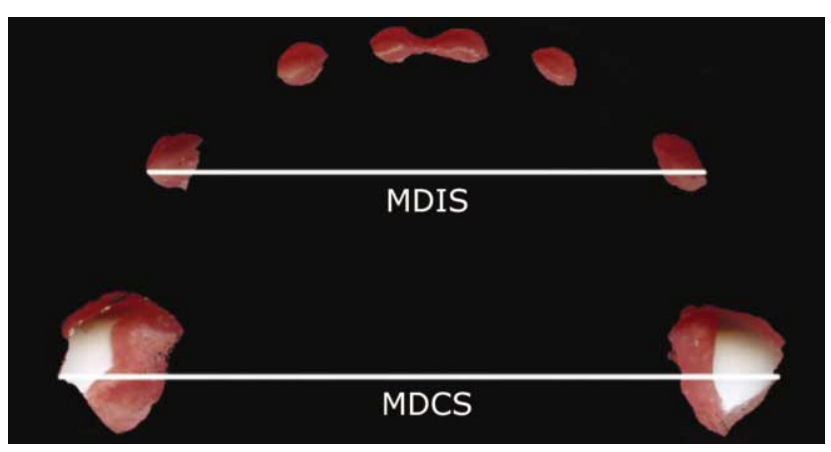

Fig. 1. Medidas odontológicas.

Análisis Estadístico. Se compararon las medidas obtenidas en los modelos de yeso, entre las tres razas estudiadas, mediante el uso de ANDEVA con diseño factorial y comparaciones múltiples; considerando un nivel de significancia de $p \leq 0,05$, y se seleccionaron aquellas variables cuantitativas que permitieron al menos una identificación intraespecie. Finalmente se realizó el estudio ciego de huellas de mordedura con el fin de identificar al menos la raza a la que pertenece la huella de mordida. Para su análisis se utilizó valores de sensibilidad, especificidad, concordancia (Kappa) y McNemar $\left(\mathrm{X}^{2}\right)$ para cada raza y con un nivel de confianza de $95 \%$.

\section{RESULTADOS}

Estudio morfométrico en modelos de yeso. En la Tabla I se presentan lo promedios y desviaciones estándar para cuatro mediciones odontológicas, para cada raza. La MDCS fue significativamente diferente entre los tres grupos, mientras que la MDCI, la MDIS) y la MDII fueron significativamente diferentes para $\mathrm{OA}$ respecto de las otras dos razas $\mathrm{p} \leq 0,05$. No se evidenció diferencias estadísticamente significativas entre sexos en las mediciones realizadas.

En relación al grado de correlación entre las mediciones efectuadas en el modelo de yeso (m) y en las huellas en cera (c) se obtuvieron los siguientes valores de correlación: MDCSm v/s MDCSc: $r=0,94 ;$ MDCI m v/s MDCIc: $\mathrm{r}=0,93$; MDISm v/s MDISc: $\mathrm{r}=0,93$ y MDIIm v/s MDIIc: $\mathrm{r}=0,9$, todos estadísticamente significativos con $\mathrm{p} \leq 0,05$. Esto permitiría consolidar la idea de la utilización de modelos de yeso de dentadura de perros como medio comparativo de una huella encontrada en un sitio de suceso, para estas mediciones.

Estudio ciego de huellas. Por causa de muerte o pérdida de algunos ejemplares se logró obtener la huella de mordedura 
TOLEDO, G. V.; IBARRA, M. L.; ROJAS, E. V.; CIOCCA, G. L.; ROCHA, D. N. \& JARA, V. G. Estudio preliminar de patrones de mordedura según forma del cráneo, mediante el análisis morfológico y morfométrico de semiarcadas dentarias de perro doméstico (Canis familiaris) con fines de identificación. Int. J. Morphol., 30(1):222-229, 2012.

Tabla I. Descripción estadística de medidas odontológicas por raza. Letras diferentes, en línea horizontal, indican diferencias significativas con $\mathrm{p} \leq 0,05$.

\begin{tabular}{lccc}
\hline Medidas odontológicas & D & B & O. A. \\
\hline MDCS (Máx. dist. canino Superior) & $\bar{X}=41,88 \mathrm{a}$ & $\bar{X}=52,7 \mathrm{~b}$ & $\bar{X}=48,13 \mathrm{c}$ \\
& $\mathrm{S}=3,55$ & $\mathrm{~S}=5,1$ & $\mathrm{~S}=2,66$ \\
MDCI (Máx. dist. canino inferior) & $\bar{X}=35,81 \mathrm{a}$ & $\bar{X}=38,53 \mathrm{a}$ & $\bar{X}=42,9 \mathrm{~b}$ \\
& $\mathrm{~S}=3,21$ & $\mathrm{~S}=5,39$ & $\mathrm{~S}=1,72$ \\
MDIS (Máx. dist. incisivo superior) & $\bar{X}=30,53 \mathrm{a}$ & $\bar{X}=32,3 \mathrm{a}$ & $\bar{X}=36,41 \mathrm{~b}$ \\
& $\mathrm{~S}=2,78$ & $\mathrm{~S}=3,07$ & $\mathrm{~S}=2,42$ \\
MDII (Máx. dist. incisivo inferior) & $\bar{X}=24,22 \mathrm{a}$ & $\bar{X}=24,69 \mathrm{a}$ & $\bar{X}=28,15 \mathrm{~b}$ \\
& $\mathrm{~S}=1,44$ & $\mathrm{~S}=3,19$ & $\mathrm{~S}=1,42$ \\
\hline
\end{tabular}

de 26 de los 30 ejemplares (86,6\%), inicialmente estudiados. El observador recibió 26 ceras con huellas de mordedura sin conocer a quien pertenece cada una de ellas. En las Tablas de Contingencia II, III y IV se comparan los resultados obtenidos por el observador (diagnóstico) y la raza y perro conocido (estándar de oro) e indican valores de sensibilidad, especificidad, concordancia (Kappa) y significancia de cambios (prueba de McNemar) para la variable MDCS y para cada raza en particular. Para las otras variables sólo se muestran los valores obtenidos.

Tabla II. Clasificación de los perros según Estándar de oro y el Diagnóstico del observador. Perros Bóxer.

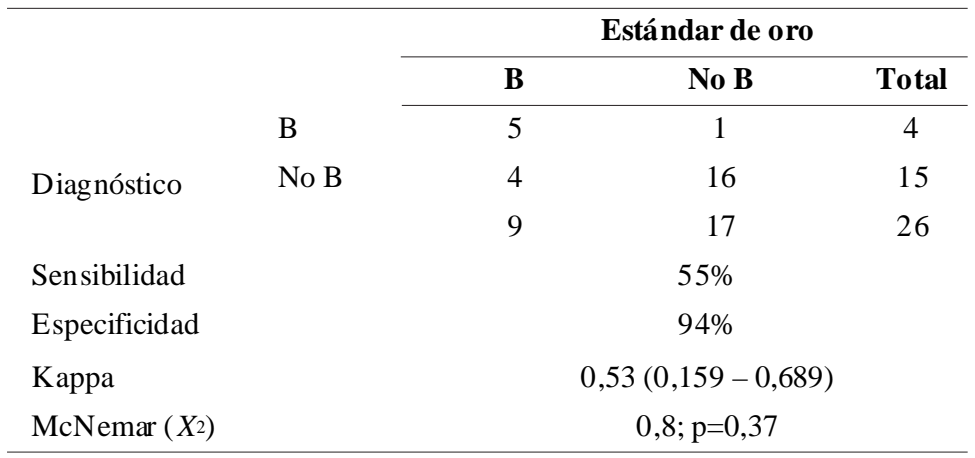

Tabla III. Clasificación de los perros según Estándar de oro y el Diagnóstico del observador. Perros Dálmata.

\begin{tabular}{llccc}
\hline & & \multicolumn{3}{c}{ Estándar de oro } \\
\cline { 3 - 5 } & & D & No D & Total \\
\cline { 3 - 5 } Diagnóstico & No D & 10 & 1 & 11 \\
& & 0 & 15 & 15 \\
Sensibilidad & 10 & 16 & 26 \\
Especificidad & & $100 \%$ & \\
Kappa & & $93 \%$ & \\
McNemar $\left(X_{2}\right)$ & & & $0,92(0,619-0,920)$ \\
\hline
\end{tabular}

MDCS (Tabla II): Esto indicaría que el $55 \%$ de los B fueron diagnosticados en el estudio ciego como tales por el observador y las otras razas, diagnosticadas correctamente como no B en un $94 \%$. El índice Kappa (Cohen) relaciona el acuerdo observado más allá del debido al azar, este calcula la diferencia entre la proporción de acuerdo observado y la proporción de acuerdo esperado por el azar. Para el diagnóstico efectuado por el observador se obtiene un valor de Kappa de 0,53 con límites entre 0,159 y 0,689 a un $95 \%$ de confianza, por lo que se puede inferir que habría concordancia estadísticamente significativa entre el observador y el estándar de oro. En el análisis para evaluar la significancia de los cambios de diagnóstico del observador respecto al estándar de oro se obtiene un valor de McNemar $\left(\mathrm{X}^{2}=0,8\right)$ con un valor de $\mathrm{p}=0,37$, por lo tanto, no significativo. Esto significa que los cambios de diagnóstico del observador respecto del estándar de oro son aleatorios por lo que no habría una tendencia en el sentido del cambio. En otras palabras, existe la misma probabilidad de considerar a un $\mathrm{B}$ como D u OA, que diagnosticar a un D u OA como B.

Para el caso del D (Tabla III) el 100\% de ellos fueron detectados como tales por el observador, mientras que las otras dos razas fueron correctamente identificadas como no D en un $93 \%$. El índice Kappa igual a 0,92 con límites de 0,619 y 0,920 , permitiría inferir que habría concordancia estadísticamente significativa en el diagnóstico dado por el observador y el estándar de oro, mediante este indicador. La prueba de McNemar $\left(\mathrm{X}^{2}=0\right)$ con un valor de $\mathrm{p}=1$, significa que los cambios de diagnóstico del observador respecto del estándar de oro son aleatorios por lo que no 
TOLEDO, G. V.; IBARRA, M. L.; ROJAS, E. V.; CIOCCA, G. L.; ROCHA, D. N. \& JARA, V. G. Estudio preliminar de patrones de mordedura según forma del cráneo, mediante el análisis morfológico y morfométrico de semiarcadas dentarias de perro doméstico (Canis familiaris) con fines de identificación. Int. J. Morphol., 30(1):222-229, 2012.

habría una tendencia en el sentido del cambio. En otras palabras, existe la misma probabilidad de considerar a un D como B u OA, que considerar a un B u OA como D.

Finalmente el $71 \%$ de los OA fueron identificados como tales por el observador y el $78 \%$ de las otras razas fueron determinadas correctamente como no OA (Tabla IV). El índice Kappa fue de 0,46 con límites de 0,077 y 0,703 infiriendo de ello una concordancia estadísticamente significativa en el diagnóstico dado por el observador y el estándar de oro, mediante este indicador. Finalmente, la prueba de $\operatorname{McNemar}\left(\mathrm{X}^{2}=0,167\right)$ con un valor de $\mathrm{p}=0,68$, como en los casos anteriores, indicaría que los cambios de diagnóstico del observador respecto del estándar de oro son aleatorios por lo que no habría una tendencia en el sentido del cambio. Es decir, existe la misma probabilidad de considerar a un OA como un $\mathrm{D}$ o $\mathrm{B}$, que considerar a un B o D como OA.

Tabla IV. Clasificación de los perros según Estándar de oro y el Diagnóstico del observador. Perros Ovejero Alemán.

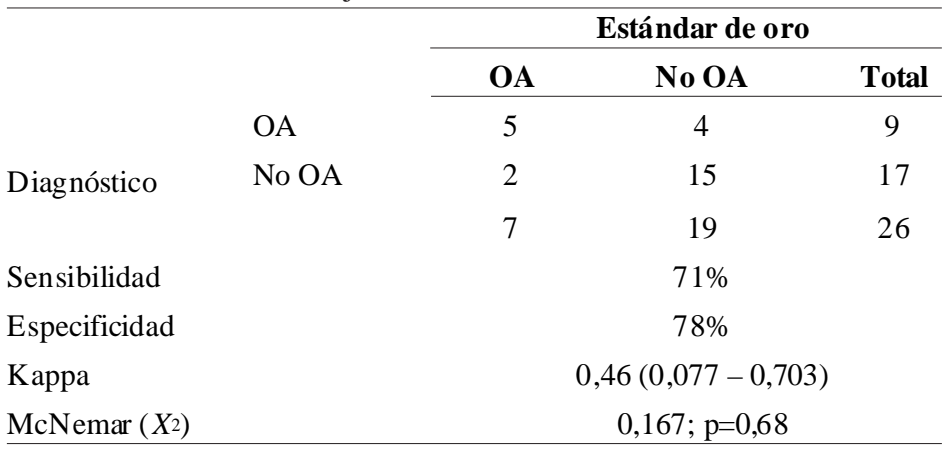

Para las otras medidas se obtuvo los siguientes resultados: MDCI: El 22\% de los perros B fueron identificados como tales por el observador mediante y las otras razas fueron diagnosticadas correctamente como no B en un 70,6\%. El $60 \%$ de los perros Dálmata fueron identificados como tales por el observador y las otras razas fueron determinadas correctamente como no dálmatas en un 70,6\%. Finalmente, el 71,4\% de los perros OA fueron diagnosticados como tales por el observador y las otras razas fueron determinadas correctamente como no OA en un 73,7 \%. Además, existiría una concordancia estadísticamente significativa entre el observador y el estándar de oro, mediante el índice Kappa para D y OA: ((k: 0,420 (0,036 a $0,697))$ y ((k: 0,397 $(0,024$ a 0,632)), respectivamente. Además, no existiría concordancia estadísticamente significativa para B ((k:$0,076\left(-0,324\right.$ a 0,302)). La prueba de $\operatorname{McNemar}\left(X^{2}=0,08\right), p=0,77$; $\left(X^{2}=0\right), p=1$ y $\left(X^{2}=0,57\right), p=0,45$, para $B, D$ y OA, respectivamente, indicaría que los cambios de diagnóstico del observador respecto del estándar de oro son aleatorios por lo que no habría una tendencia en el sentido del cambio.

MDIS: El 71,4 \% de los B fueron identificados como tales por el observador y las otras razas fueron determinadas correctamente como no B en un 73,7 \%. Por otro lado, el $80 \%$ de los perros
Dálmata fueron identificados como tales por el observador y las otras razas fueron diagnosticadas correctamente como no D en un 93,8 \%. Finalmente el $71,4 \%$ de los perros OA fueron identificados como tales por el observador y las otras razas fueron diagnosticadas correctamente como no OA en un $63,2 \%$. Por otro lado, existiría concordancia estadísticamente significativa entre el observador y el estándar de oro, mediante el índice Kappa para B y D: ((k:0,397 (0,024 a 0,632)) y $((\mathrm{k}: 0,752(0,389$ a 0,884$)$ ), respectivamente. Sin embargo no existiría concordancia estadísticamente significativa para OA ((k: 0,282 (- 0,066 a 0,502)).

La prueba de $\mathrm{McNemar}\left(\mathrm{X}^{2}=1,5\right), \mathrm{p}=0,22$; $\left(\mathrm{X}^{2}=0\right), \mathrm{p}=1 \mathrm{y}\left(\mathrm{X}^{2}=1,78\right), \mathrm{p}=0,18$, para $\mathrm{B}, \mathrm{D} \mathrm{y}$ $\mathrm{OA}$, respectivamente, indicaría que los cambios de diagnóstico del observador respecto del estándar de oro son aleatorios por lo que no habría una tendencia en el sentido del cambio.

MDII: El 22,2 \% de los B fueron identificados como tales por el observador y las otras razas fueron diagnosticadas correctamente como no B en un $70,6 \%$. El $60 \%$ de los perros D fueron identificados como tales por el observador mientras que las otras razas fueron diagnosticadas correctamente como no D en un $81,3 \%$. Finalmente el $85,7 \%$ de los perros $\mathrm{OA}$ fueron diagnosticados como tales por el observador y las otras razas fueron determinadas correctamente como no OA en un 81,3\%. Existiría concordancia estadísticamente significativa entre el observador y el estándar de oro, mediante el indicador Kappa para D y OA: ((k: 0,42 (0,036 a 0,697)) y $((\mathrm{k}: 0,57(0,196$ a 0,709$))$ respectivamente, mientras que para B no existiría tal concordancia ( $(\mathrm{k}$ :0,076 (- 0,324 a 0,302)). La prueba de McNemar $\left(\mathrm{X}^{2}=0,083\right), \mathrm{p}=0,77 ;\left(\mathrm{X}^{2}=0\right), \mathrm{p}=1 \mathrm{y}\left(\mathrm{X}^{2}=0,8\right)$, $\mathrm{p}=0,37$, para B, D y OA, respectivamente, indicaría que los cambios de diagnóstico del observador respecto del estándar de oro son aleatorios por lo que no habría una tendencia en el sentido del cambio.

Estudio morfológico: En las Figuras 2, 3 y 4 se evidencian las características morfológicas de la huella de mordedura en cera de B, D y OA, respectivamente. Se observa un mayor número de incisivos y mayor ancho de la semiarcada superior en B.

Al realizar el estudio individual de la disposición de cada diente, su angulación y forma (en la huella de mordedura) se logró identificar e individualizar, en el estudio ciego, al $100 \%$ de los ejemplares. 


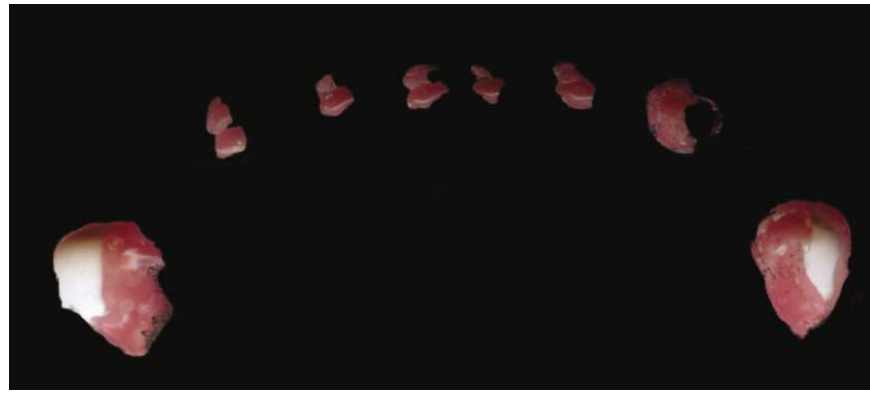

Fig. 2. Semiarcada superior de Bóxer.

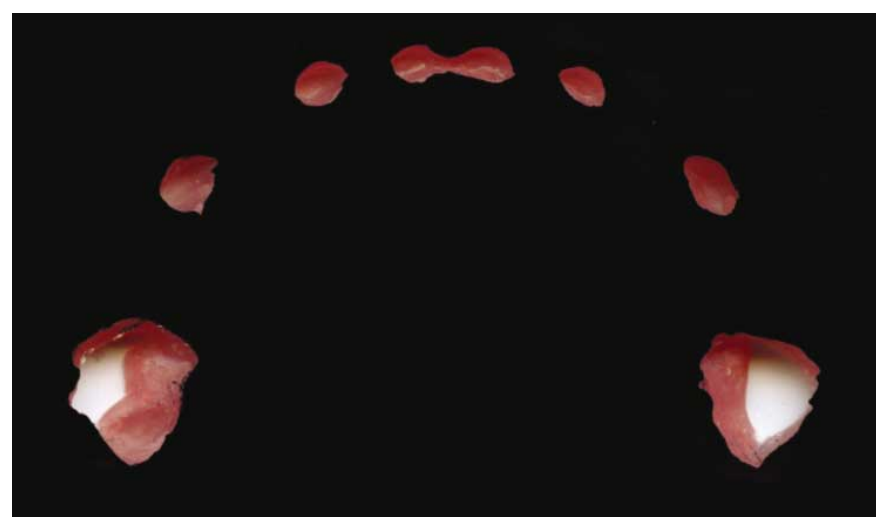

Fig. 3. Semiarcada superior de Dálmata.

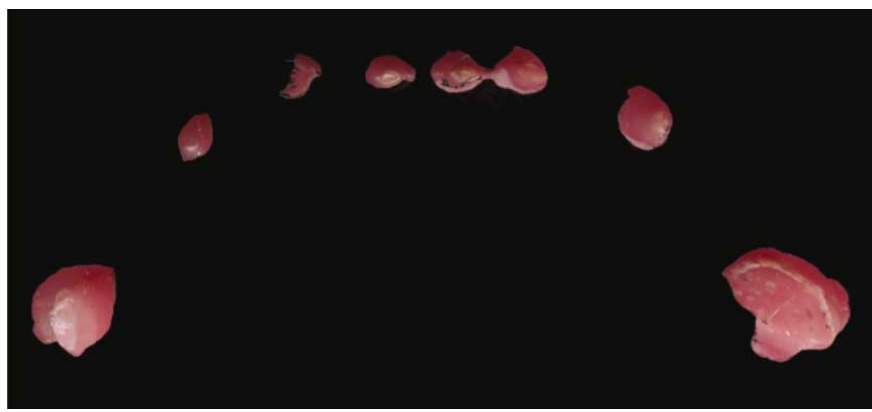

Fig. 4. Semiarcada superior de Ovejero Alemán.

\section{DISCUSIÓN}

Para la identificación de agresores humanos, mediante la dentadura, se acude a la elaboración de réplicas de éstas utilizando técnicas ampliamente difundidas y que requieren materiales odontológicos como el alginato, para la impresión de la dentadura del sospechoso y el yeso para el vaciado de modelos. Si bien la utilización de alginato y yeso no fue un problema en este estudio, cabe señalar que, algunos autores sugieren la utilización de otros materiales como el vinilopolixiloxano (silicona) para la impresión, y el plástico o resinas para el vaciado, proporcionando una mejor calidad y durabilidad a diferencia del alginato que genera impresiones no reutilizables y que debe ser procesado en el instante, mientras que el yeso puede provo- car la existencia de burbujas incrementando la posibilidad de fracturas del modelo (Gaensslen). Dado que en Chile la casuística de ataques mortales por causa de perros es de baja incidencia (Departamento de Estadística del Servicio Médico Legal) a diferencia de aquellos casos no mortales reportados en Servicios de Salud Metropolitano, sería económicamente posible implementar sistemas de mayor costo y calidad. Sin embargo, y considerando el número de ejemplares utilizados en este estudio, igualmente fue posible cumplir el objetivo de identificación de la raza utilizando materiales de menor costo $\mathrm{y}$ factibilidad operativa. El bajo costo que representa este procedimiento, permitiría su utilización en el SS, considerando el alto número de casos de mordidas en Santiago (Ibarra et al.). En los modelos de yeso fue posible determinar y comparar distintas medidas odontológicas. Sólo una de estas medidas permitió diferenciar e identificar, estadísticamente, la raza: MDCS ( $\overline{\mathrm{x}}: 41,88 \mathrm{~mm} ; 48,13 \mathrm{~mm}$ y $52,7 \mathrm{~mm}$ para D, OA y B, respectivamente). El resto de las mediciones sólo permite diferenciar una raza respecto de las otras dos. En este contexto cabe señalar el estudio de otros autores, que determinan parámetros morfométricos de dentición animal con el fin de identificar diferentes especies animales, pero en ningún caso diferenciar e identificar distintas razas de perros (Murmann et al., 2006). Se considera la MDCS a diferencia de las mediciones propuestas por Murmann, debido a que en una herida se hace dificultoso y poco preciso estimar el punto exacto de entrada (cúspide dentaria). Sin embargo, es más factible determinar y realizar mediciones a través de los bordes externos de las piezas dentarias que en teoría debieran coincidir con aquellos bordes externos de una herida determinada. Cabe destacar que para este estudio se han considerado ejemplares de raza pura. Esto permitiría reducir cualquier factor adicional (ej. error por mestizaje) que pudiese afectar las mediciones antes descritas. Conjuntamente se desprende de los análisis que ninguna de las mediciones logró diferenciar por sexo, pero si se logró individualizar, en parte, a la raza del supuesto agresor, lo que indicaría la necesidad de realizar análisis morfológicos que aborden el estudio de características individuales de su dentadura.

La alta correlación existente entre las mediciones realizadas en los modelos de yeso y las huellas de mordida permitiría utilizar estos modelos como un medio de fijación de la dentadura de un ejemplar al momento de la agresión o de hallazgo de un cadáver. Sin embargo, los valores obtenidos permitirían al menos acotar el espectro de búsqueda entregando una probabilidad de coincidencia. Pretty \& Sweet (2001), señalan que no hay un mínimo número de puntos de concor- 
TOLEDO, G. V.; IBARRA, M. L.; ROJAS, E. V.; CIOCCA, G. L.; ROCHA, D. N. \& JARA, V. G. Estudio preliminar de patrones de mordedura según forma del cráneo, mediante el análisis morfológico y morfométrico de semiarcadas dentarias de perro doméstico (Canis familiaris) con fines de identificación. Int. J. Morphol., 30(1):222-229, 2012.

dancia o características que son requeridas para una identificación positiva. En muchos casos, un simple diente puede ser utilizado para la identificación si éste contiene suficientes características únicas. En este contexto se logró identificar e individualizar, mediante el análisis ciego, al 100\% de los animales (n:26) a través de las características de su huella de mordedura en cera, contemplando las características individuales de los dientes en estudio, lo que refuerza la necesidad de hacer estudios morfológicos complementarios a los morfométricos para la identificación y más importante aún la individualización de cada ejemplar.

A pesar de cumplir el objetivo de identificar e individualizar cada ejemplar mediante su huella de mordedura, es necesario recordar que las mordeduras de perros pueden ser múltiples, con otros componentes asociados como laceración, abrasión y contusión-hematoma (Gaensslen).
Sin duda la promulgación de la futura ley de tenencia responsable dará un gran impulso a estudios en este contexto abordando un mayor número de perros y razas, en particular las denominadas potencialmente peligrosas. Dentro de sus disposiciones debería contemplar un registro amplio para cada mascota, identificando a un perro asociado a un dueño, indicando algunas características propias de cada animal (características dentarias, malformaciones de piezas dentales (giroversión, ausencia), patrón de pelaje, manchas, etc.) como ocurre con otras especies de alto valor económico.

AGRADECIMIENTOS. Dr. Ricardo Olivares P-M. Académico Unidad de Anatomía Facultad de Ciencias Veterinarias y Pecuarias. Universidad de Chile; Escuela de Adiestramiento Canino, Carabineros de Chile y Centro de Adiestramiento Canino Dialma, Chicureo, Chile.

TOLEDO, G. V.; IBARRA, M. L.; ROJAS, E. V.; CIOCCA, G. L.; ROCHA, D. N. \& JARA, V. G. Preliminary study of bite patterns according skull shape, by morphological and morphometric analysis of dental semi arcs of domestic dog (Canis familiaris) for identification. Int. J. Morphol., 30(1):222-229, 2012.

SUMMARY: Forensic dentistry is a branch of Forensic Sciences used to establish the identity of a crime victim or perpetrator, among these studies are bite marks and dental chronological age studies. In the absence of such studies in Chile and considering the widespread problem of dog bites in Chile, bite patterns were compared according to skull shape, by morphological and morphometric analysis of dental semi arcs of domestic dog (Canis Familiaris) for medico legal purposes. Plaster dental models and impressions of bite marks were obtained of three pure breeds: Boxer, Dalmatian and German shepherd. Measures included he maximum external distance between upper and lower canine, and maximum external distance between upper and lower incisors. Main results show that only the maximum distance between upper canine would allow statistically significant race identification, $(\mathrm{p} \leq 0.05)$ in cast models. In a blind study and from a morphometric point of view, Dalmatians were identified with a high rate of sensitivity and specificity. A somewhat lesser and variable form was observed in Boxer and German Shepherd. The morphological study allowed identification and individualization of $100 \%$ of dogs. Therefore, both morphological and morphometric methods are useful and complementary tools to identify and individualize a potentially aggressive dog.

KEY WORDS: Dog bit; Bite pattern; Forensic veterinary.

\section{REFERENCIAS BIBLIOGRÁFICAS}

Anusavice, K. J. Phillips ciencia de los materiales dentales. 11a Ed. Madrid, Elsevier, 2004.

Bowers, C. M. \& Bell, G. L. Manual of forensic odontology. 3rd ed. Montpelier, Printing Specialists, 1995.

Ciocca, L. Huellas o marcas de mordida. Su valor criminalístico y legal. Odontol. Chil., 35:142-50, 1987.

Ciocca, L. Odontología Médico-Legal. Santiago, Ed. Jurídicas de Santiago, 2010. p.550.

David, T. J. Adjunctive use of scanning electron microscopy in bite mark analysis: a three-dimensional study. J. Forensic Sci., 31:1126-34, 1986.

Drinnan, A. J. \& Melton, M. J. Court presentation of bitemark evidence. Int. Dent. J., 35:316-21, 1985.
Gaensslen. Bitemark Evidence. New York, Marcel Dekker; 2005. pp.293-321.

Ibarra, L.; Morales, M. A. \& Cáceres, L. Mordeduras a personas por ataque de perros en la ciudad de Santiago, Chile. Av. Cs. Vet. 18:41-6, 2003.

Jakobsen, J.; Holmen, L.; Fredebo, L. \& Sejrsen, B. Scanning electron microscopy, a useful tool in forensic dental work. $J$. Forensic Odontostomatol., 2:36-40, 1995.

Manns, A. Sistema estomatognático. Santiago, Facultad de Odontología, Universidad de Chile, 1988. pp.75-84.

McNamee, A. H.; Sweet, D. \& Pretty, I. A comparative reliability analysis of computer-generated bite mark overlays. J. Forensic Sci., 50:400-5, 2005. 
TOLEDO, G. V.; IBARRA, M. L.; ROJAS, E. V.; CIOCCA, G. L.; ROCHA, D. N. \& JARA, V. G. Estudio preliminar de patrones de mordedura según forma del cráneo, mediante el análisis morfológico y morfométrico de semiarcadas dentarias de perro doméstico (Canis familiaris) con fines de identificación. Int. J. Morphol., 30(1):222-229, 2012.

Morgan, M. The microbiology of animal bites. Bul. Roy. Coll. Pathol., 28:16-9, 2004.

Morgan, M. \& Palmer, J. Dog Bites. BMJ, 24:413-7, 2007.

Murmann, D.; Brumit, P. \& Schrader, B. A Comparison of Animal Jaws and Bite Mark Patterns. J. Forensic Sci., 51:846-60, 2006.

Palacio, J.; León, M. \& García-Belenguerb, S. Aspectos epidemiológicos de las mordeduras caninas. Gac. Sanit. 19:50$8,2005$.

Pretty, I. A. \& Sweet, L. D. A look at forensic dentistry - Part 2: Teeth as weapons of violence - identification of bitemark perpetrators. Br. Dent. J., 190:415-8, 2001.

Rawson, R. D.; Bell, A.; Kinard, B. S. \& Kinard, J. G. Radiographic interpretation of contrast-media-enhanced bite marks. $J$. Forensic Sci., 24:898-901, 1979.

Sweet, D.; Lorente, M.; Lorente, J. A.; Valenzuela, A. \& Villanueva, E. An improved method to recover saliva from human skin: the double swab technique. J. Forensic Sci., 42:320-2, 1997.

Sweet, D. \& Bowers, C. M. Accuracy of bitemark overlays: a comparison of five common methods to produce exemplars from a suspect's dentition. J. Forensic Sci., 43:362-7, 1998.

Teke, A. Odontología Legal. 2a. Ed. Santiago, Editorial Mediterráneo, 2001. pp.229-58.

\author{
Dirección para correspondencia: \\ Víctor Toledo González \\ Facultad de Ciencias Veterinarias y Pecuarias \\ Universidad de Chile \\ Casilla 2 correo 15 \\ La Granja \\ Santiago \\ CHILE
}

Email: vtoledog@gmail.com

Recibido : 01-08-2011

Aceptado: 22-10-2011 